\title{
ATHENA X-ray Integral Field Unit on- board event processor: analysis of performance of two triggering algorithms
}

Beatriz Cobo, María Teresa Ceballos, Philippe Peille, Jean-Michel Mesnager, Joern Wilms, et al.

Beatriz Cobo, María Teresa Ceballos, Philippe Peille, Jean-Michel Mesnager, Joern Wilms, Thomas Dauser, Christian Kirsch, Frédéric Pinsard, Christophe Cara, Michel Gros, Laurent Ravera, Simon Bandler, Frederick Porter, Caroline Kilbourne, Kazuhiro Sakai, "ATHENA X-ray Integral Field Unit on-board event processor: analysis of performance of two triggering algorithms," Proc. SPIE 10699, Space Telescopes and Instrumentation 2018: Ultraviolet to Gamma Ray, 106994 S (6 July 2018); doi: $10.1117 / 12.2313268$

EDIE Event: SPIE Astronomical Telescopes + Instrumentation, 2018, Austin, Texas, United States 


\title{
Athena X-ray Integral Field Unit on-board Event Processor: Analysis of performance of two triggering algorithms
}

\author{
Beatriz Cobo ${ }^{\mathrm{a}}$, María Teresa Ceballos ${ }^{\mathrm{a}}$, Philippe Peille ${ }^{\mathrm{b}}$, Jean-Michel Mesnager ${ }^{\mathrm{b}}$, Joern \\ Wilms ${ }^{c}$, Thomas Dauser ${ }^{\mathrm{c}}$, Christian Kirsch ${ }^{\mathrm{c}}$, Frederic Pinsard ${ }^{\mathrm{d}}$, Christophe Cara ${ }^{\mathrm{d}}$, Michel \\ Gros $^{\mathrm{d}}$, Laurent Ravera ${ }^{\mathrm{e}}$, Simon Bandler ${ }^{\mathrm{f}}$, Frederic Porter $^{\mathrm{f}}$, Caroline Kilbourne ${ }^{\mathrm{f}}$, and Kazuhiro \\ Sakaif \\ anstituto de Física de Cantabria (CSIC-UC), Edificio Juan Jordá, Avenida de los Castros, s/n \\ - E-39005 Santander, Cantabria, Spain \\ ${ }^{\mathrm{b}}$ CNES, 18 Av. Edouard Belin, 31401 Toulouse Dedex 9, France \\ ${ }^{\mathrm{c}}$ ECAP, University of Erlangen-Nuremberg, Sternwartstr. 7, 96049 Bamberg, Germany \\ ${ }^{\mathrm{d} C E A}$ Saclay, Bat. 709 Orme de Merisiers, 91191 Gif-sur-Yvette, France \\ eIRAP CNRS, 9 Av. Colonel Roche, BP 44346, F-31028 Toulouse Cedex 4, France \\ ${ }^{\mathrm{f}}$ NASA Goddard Space Flight Center, 8800 Greenbelt Road, Greenbelt, Maryland 207701, USA
}

\begin{abstract}
In the framework of the ESA Athena mission, the X-ray Integral Field Unit (X-IFU) micro-calorimeter will provide unprecedented spatially resolved high-resolution X-ray spectroscopy. For this purpose, the on-board Event Processor (EP) must initially trigger the current pulses induced by the X-ray photons hitting the detector to proceed with a reconstruction which provides the arrival time, spatial location and energy of each event. The current event triggering design is implemented in two stages: one initial trigger of the low-pass filtered derivative of the raw data to extract records containing pulses and a second stage performing a fine detection to look for all the pulses in the record. In order to establish the current baseline detection technique of the EP in the $\mathrm{X}$-IFU instrument, an assessment of the capabilities of different triggering algorithms is required, both in terms of performance (detection efficiency) and computational load, as processing will take place on-board. We present a comparison of two detection algorithms, the Simplest Threshold Crossing (STC) and the model-dependent Adjusted Derivative (AD). The analysis also evaluates the (possible) negative effect of different instrumental scenarios as a reduced sampling rate. The evaluations point out that the simplest algorithm STC shows worse performance than $\mathrm{AD}$ for the smallest pulses separations and the lowest secondary energies. Nevertheless, checking the expected number of such pulses combinations in a typical bright source observation, we conclude that it does not have impact in the science. Moreover, the savings in the computational resources and calibration needs make STC a valuable option.
\end{abstract}

Keywords: Athena, X-ray Integral Field Unit, X-ray spectroscopy, space telescopes, instrumentation, Event Processor, pulse detection, performance analysis

\section{INTRODUCTION}

The X-ray Integral Field Unit $(\mathrm{X}-\mathrm{IFU})^{1}$ instrument that will be on-board the ESA's Athena mission, ${ }^{2}$ is a high-resolution cryogenic imaging spectrometer in the $0.2-12 \mathrm{keV}$ band that will provide unprecedented spectral resolution $(2.5 \mathrm{eV}$ at $7 \mathrm{keV})$ with 5 " of spatial resolution. The X-IFU Focal Plane will contain an array of 3840 Transition Edge Sensors (TES) in groups of 40 TES per readout channel. After demodulating the readout chains, the on-board Event Processor $(\mathrm{EP})^{3}$ performs a reconstruction job in two stages, the triggering of the $\mathrm{X}$-ray pulses and the reconstruction of the energy, arrival time and spatial location of the detected events.

Further author information: (Send correspondence to Beatriz Cobo)

Beatriz Cobo: E-mail: cobo@ifca.unican.es

Space Telescopes and Instrumentation 2018: Ultraviolet to Gamma Ray, edited by Jan-Willem A. den Herder, Shouleh Nikzad, Kazuhiro Nakazawa, Proc. of SPIE Vol. 10699, 106994S · @ 2018 SPIE CCC code: $0277-786 X / 18 / \$ 18 \cdot$ doi: $10.1117 / 12.2313268$ 
An initial trigger is done in hardware, where the processor selects the chunks of raw data streams which contain pulses, the pulse records, in order to limit the amount of data sent to the second phase, where the reconstruction software extracts the pulses parameters. The first step to be performed during reconstruction is the search for additional events in the initially triggered record (more than one pulse is possible). After this, the software estimates the energy and the arrival time of each individual event.

A performance assessment of different pulse reconstruction algorithms ${ }^{4}$ led to the selection of the optimal filtering technique ${ }^{5-7}$ applied to a transformed quasi-resistance space ${ }^{8}$ as the current baseline* mechanism for the X-IFU event reconstruction, since it resulted in the best compromise between energy resolution and computational and calibration costs.

Similarly, in this paper we present a comparative analysis of two detection algorithms, the Adjusted Derivative and the Single Threshold Crossing, selected to perform the fine triggering of the EP-Event Reconstruction. These methods are applied to synthetic data streams containing one pair of events per record, spanning through a range of energies and separations for the primary (first) and secondary (second) pulses in each pair. In Sec. 2 we describe the details of the simulations performed for the analysis. A description of the triggering algorithms is done in Sec. 3, and Sec. 4 presents a complementary flagging algorithm for those cases where detection fails.

The different capabilities of the two methods are reviewed in terms of the detection efficiency (in Sec. 5), the frequency of occurrence of the conflictive events (in Sec. 6) and the computational load (Sec. 7). Finally, The consequences of reducing the number of available templates or the sampling rate will be shown in Sec. 8.

\section{TEST DATA SIMULATION}

The data used in this analysis have been simulated with the SIXTE end-to-end simulator tool tessim ${ }^{9}$ using the pixel parameters of Tab. 1, in order to get representative X-IFU pixels. ${ }^{10}$ One thousand pairs of photons have been simulated at each combination of primary and secondary energies in the interval $0.2-8 \mathrm{keV}$, and separations ranging from $25.6 \mu$ s to $12.8 \mathrm{~ms}$. Photons have been placed at a random phase with the sampling rate to mimic the behavior of the jitter (the random offset between the actual pulse arrival times and the trigger times) in the real data.

Table 1. Pixel parameters used to simulate the baseline X-IFU pixels (LPA2shunt).

\begin{tabular}{|l|l|}
\hline Pixel parameter & Value \\
\hline Heat capacity at bias C & $0.8 \mathrm{pJ} / \mathrm{K}$ \\
Bath conductance at bias & $115 \mathrm{pW} / \mathrm{K}$ \\
Heat bath power flow exponent & 3 \\
$\alpha$ & 75 \\
$\beta$ & 1.25 \\
Bias resistance $R_{0}$ & $1 \mathrm{~m} \Omega$ \\
Bias temperature $T_{0}$ & $90 \mathrm{mK}$ \\
Bias current $I_{0}$ & $51.6 \mu \mathrm{A}$ \\
Effective load resistance $R_{L}$ & $60 \mu \Omega$ \\
Effective circuit inductance $\mathrm{L}$ & $121 \mathrm{nH}$ \\
\hline
\end{tabular}

\footnotetext{
${ }^{*}$ For this study the current space is used for simplicity.

${ }^{\dagger}$ The baseline sampling rate is $156.25 \mathrm{kHz}$.
} 


\section{PRESENTATION OF THE DIFFERENT TRIGGERING ALGORITHMS}

\subsection{Adjusted Derivative (AD) algorithm}

In the $\mathrm{AD}$ detection method ${ }^{7}$ (Fig. 1), pulses are detected when a sample of the signal derivative passes a pre-defined threshold ${ }^{\ddagger}$ and this point marks the (initial) start time of an event. Based on this specific value of the derivative signal, a template is selected from the on-the-ground pre-computed template library to perform a 25-samples-long dot product of the pre-detected pulse and the template. This dot product is then calculated at different positions around the initial starting time of the pulse to better determine the correct starting point (maximum value of the product). Based on this arrival time a new template, interpolated between the templates embracing the new derivative value, is selected again. Then the 100-samples long template is subtracted and the residual signal searched recursively for new pulses until no additional events are found.

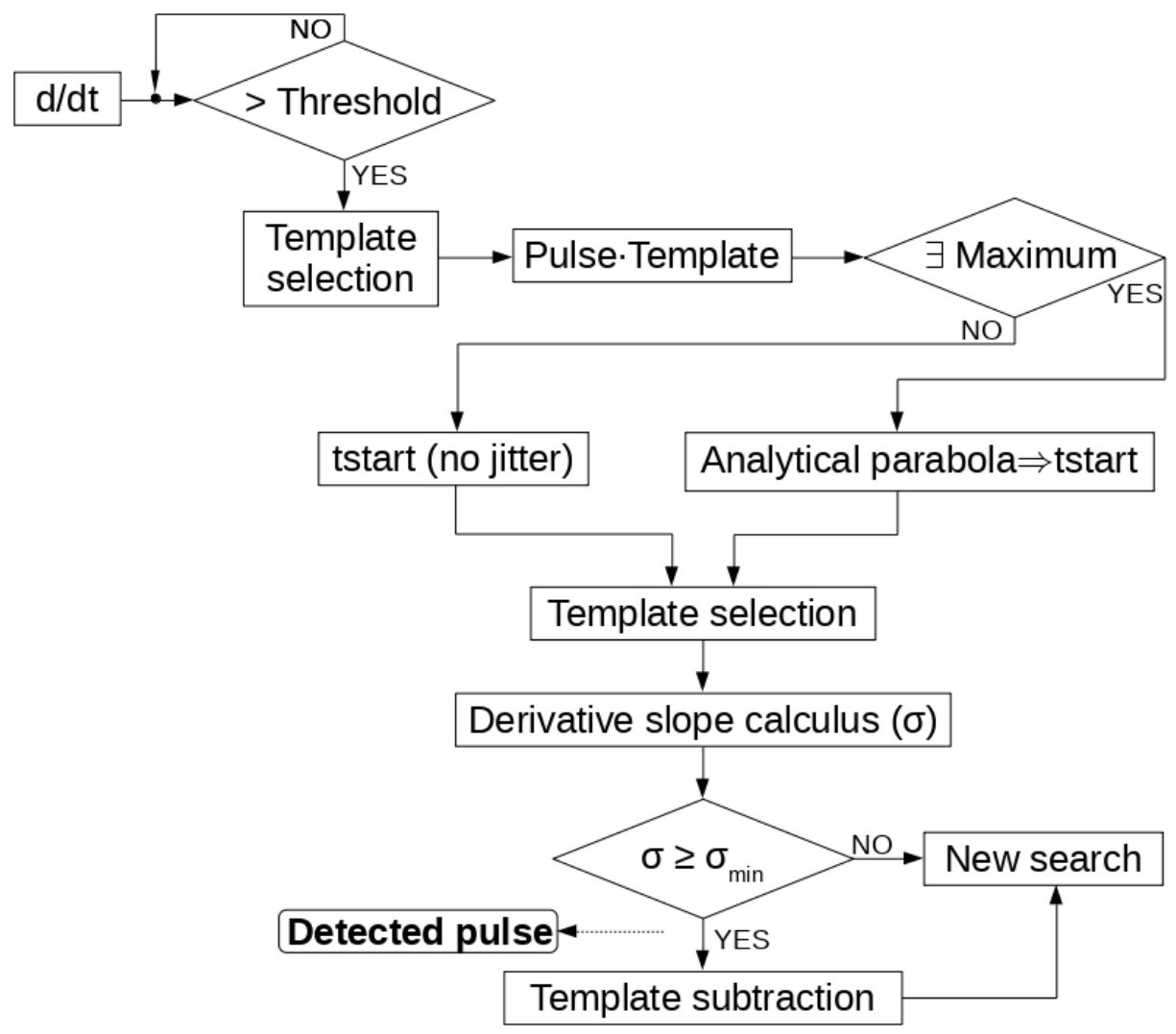

Figure 1. Block diagram explaining the AD detection process

Usually a dot product in 3 different lags around the sample of the initial detection is adequate to find a maximum $^{\S}$ and the following steps in the algorithm will depend on whether a maximum of the dot product has been found or not:

- If a maximum of the dot product has not been found, the starting time of the pulse is fixed to the time when the derivative gets over the threshold (in this case, the starting time of the pulse matches a digitized sample without taking the possible jitter into account).

\footnotetext{
${ }^{\ddagger}$ To determine the threshold level, a pulse-free stream is differentiated and a median-kappa-clipping process is applied to the data. The threshold used in the current study is the best compromise to detect the smallest pulses that could be received ( $0.2 \mathrm{keV}$ according to the X-IFU specifications) and to avoid the detection of residuals in the signal.

${ }^{\S}$ Nevertheless, when the residual signals are large, the maximum of the dot product moves towards the secondary pulse, missing the primary detection. This is why currently the maximum number of the dot product lags is limited to 5 .
} 
- If a maximum of the dot product has been found, a new starting time of the pulse is established (by using the 3-dot-product results around the maximum to analytically define a parabola and locate its maximum). Then, an iterative process ${ }^{\mathbb{I}}$ begins to select the best template from the library, resulting each time in a new starting time with a different jitter.

The pulse template subtraction for close pulses of large energies usually leaves large residuals that could be detected as new events. To avoid this behavior, an additional check using the slope of the differentiated signal marked by 3 consecutive samples $\left(T_{\text {start }}-1, T_{\text {start }}, T_{\text {start }}+1\right)$ is performed after the pulse relocation and compared to a reference value (the slope of the smallest expected pulses derivative). A potential event whose slope is lower than this value is thus discarded (it is likely a residual signal) and a new search starts. If the slope is higher than the minimum slope of the templates in the calibration library, the pulse is considered as a detection.

\subsection{Single Threshold Crossing (STC) algorithm}

This is the simplest algorithm (represented in Fig. 2) which relies only in the signal trespassing a given threshold and thus, no library of templates is required. Due to the noise in the signal, the threshold criteria must involve several (predetermined) samples of the derivative. When they are above the threshold a pulse is detected. After the detection, the first sample that went above the threshold is taken as the start time of the detected events. The triggering process continues only if a certain number of samples of the derivative comes back again below the threshold.

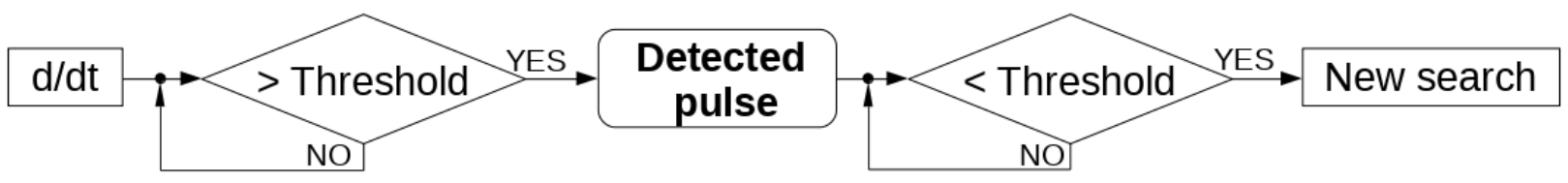

Figure 2. Block diagram explaining the STC detection process

\section{FLAGGING SCHEME FOR PILED-UP PULSES}

When two events are close enough, both detection algorithms (STC and AD) can fail trying to detect every single pulse, because of a derivative signal continuously above the selected threshold (STC algorithm) or because the remaining signal after a primary subtraction does not pass the detection criteria (AD algorithm).

For this reason, a flagging scheme is needed to mark these pulses as piled-up. Such a mechanism could be a low resolution estimation of the energy of the pulse(s) as for example, the mean value of the four initial points of the derivative of the primary (detected) pulse. The comparison of this value with the equivalent in the templates library should give an idea of the expected reconstructed energy (if a single pulse were involved). Any large deviation from this value would thus point to a possible pile-up.

Bagplots (see Fig. 3 as an example) can be used to represent the reconstructed energy versus the mean of the 4 initial samples of the derivative. The area where reconstructed single pulses, simulated at the energy of the primary events in the pairs, are located is plotted in the yellow bagplot whereas the blue-colored bagplots locate 1000 tessim-simulated pairs of pulses which have been reconstructed as a single pulse (mimicking a failure in the secondary detection). Reconstruction of pairs (blue) and single pulses (yellow) is done with a High resolution optimal filter" built from a $6 \mathrm{keV}$ template.

As it can be seen for this specific example, when the secondary pulses are at a distance of around $2.1 \mathrm{~ms}$ (325-330 samples) from the primary ones, the resulting piled-up pulses cannot be distinguished (in the energy

\footnotetext{
This process stops when the selected template is the same as in the previous iteration or when there are no more templates in the library.

"Currently and for the baseline sampling frequency, a HighRes filter is a 8192-samples-length filter, a MidRes filter is a 512-samples-length filter and a LimRes filter is a 256-samples length filter.
} 
vs. derivative space) from the single pulses at the energy of the primary pulse in the couple. In these cases, the pair will not be flagged as piled-up but will be treated as a normal single pulse without being noticed whereas it is indeed a pile-up event.

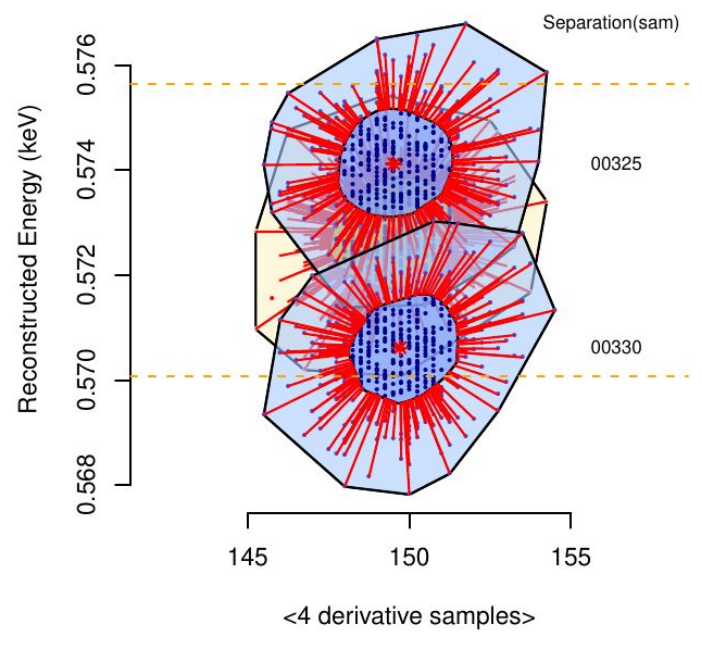

Figure 3. Bagplots corresponding to 1000 pairs of pulses with primary energies of $E_{P}=0.5 \mathrm{keV}$ and secondary energies of $E_{S}=2 \mathrm{keV}$. The pulses separation is given on the right labels in samples. Orange horizontal lines enclose the area where single and double pulses will be confused (see text for details).

Fig. 4 (corresponding to the same example case for the previous bagplots) represents the mean energy of the pairs of pulses as a function of the separation between the pulses. Pairs are reconstructed as single pulses using a High resolution filter. Each point shows the mean value of the reconstructed energy of the 1000 pairs (error bars are inappreciable). Vertical gray lines determine the separations for which piled-up and single pulses would be confused.

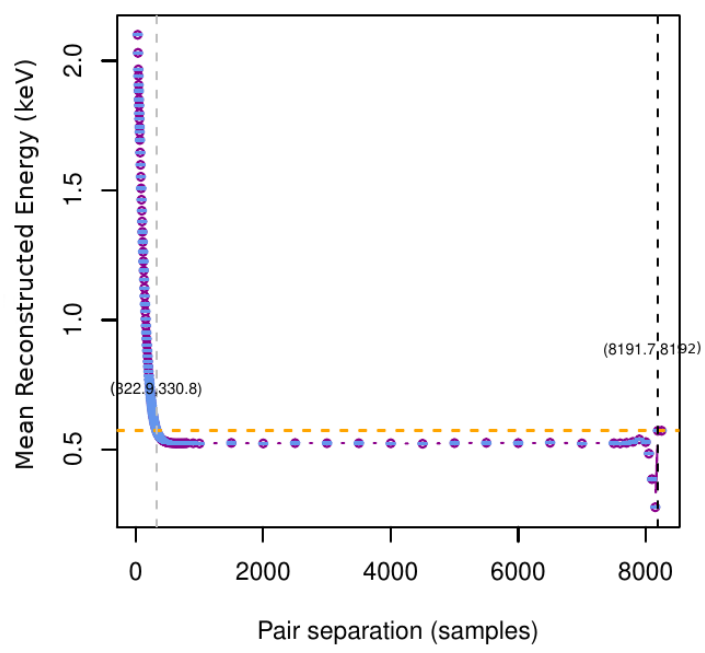

Figure 4. Reconstructed energy of 1000 pairs of pulses with primary energies of $E_{P}=0.5 \mathrm{keV}$ and secondary energies of $E_{S}=2 \mathrm{keV}$. Orange horizontal lines enclose the area where single and double pulses will be confused. Vertical dashed lines mark the intersection of the reconstruction curve with the orange conflict area. See text for details. 


\section{DETECTION PERFORMANCE}

In order to compare the detection performance between the AD detection and the STC algorithms, two groups of colored maps have been built. The detection maps give information about the lost pulses in the detection process and the fake maps give information about the positive-false detected pulses. If only the total number of detected pulses were analyzed, the added effect of failed and false detection could mimic the perfect detection.

\subsection{Detection maps}

Every plot represents the detection performance for pairs of pulses with a given primary energy. The $\mathrm{X}$ axis represents the energy of the secondary pulse (keV) and the $\mathrm{Y}$ axis the separation of the pulses (ms). Cells give the percentage of detection (white numbers correspond to percentages of exactly 100\%). The boxes in fluorescent colors represent the areas from the bagplots analysis where pile-up (secondary undetection) cannot be distinguished from single pulses at different reconstruction filter lengths (yellow for HighRes filter, cyan for MidRes filter and green for LimRes filter). Circles are used to help with the visualization of the smallest boxes.

As it can be seen in the map representing the $E_{P}=0.2 \mathrm{keV}$ (left plot in Fig. 5) for the AD method, in spite of the greenish colors in most of the cells, the detection is never perfect (always different from 100\%) because the criteria of the slope of the derivative is hard to achieve by the smallest primary pulses. In the STC case the primaries detection probability is always perfect, for whatever separation and secondary pulse energy.
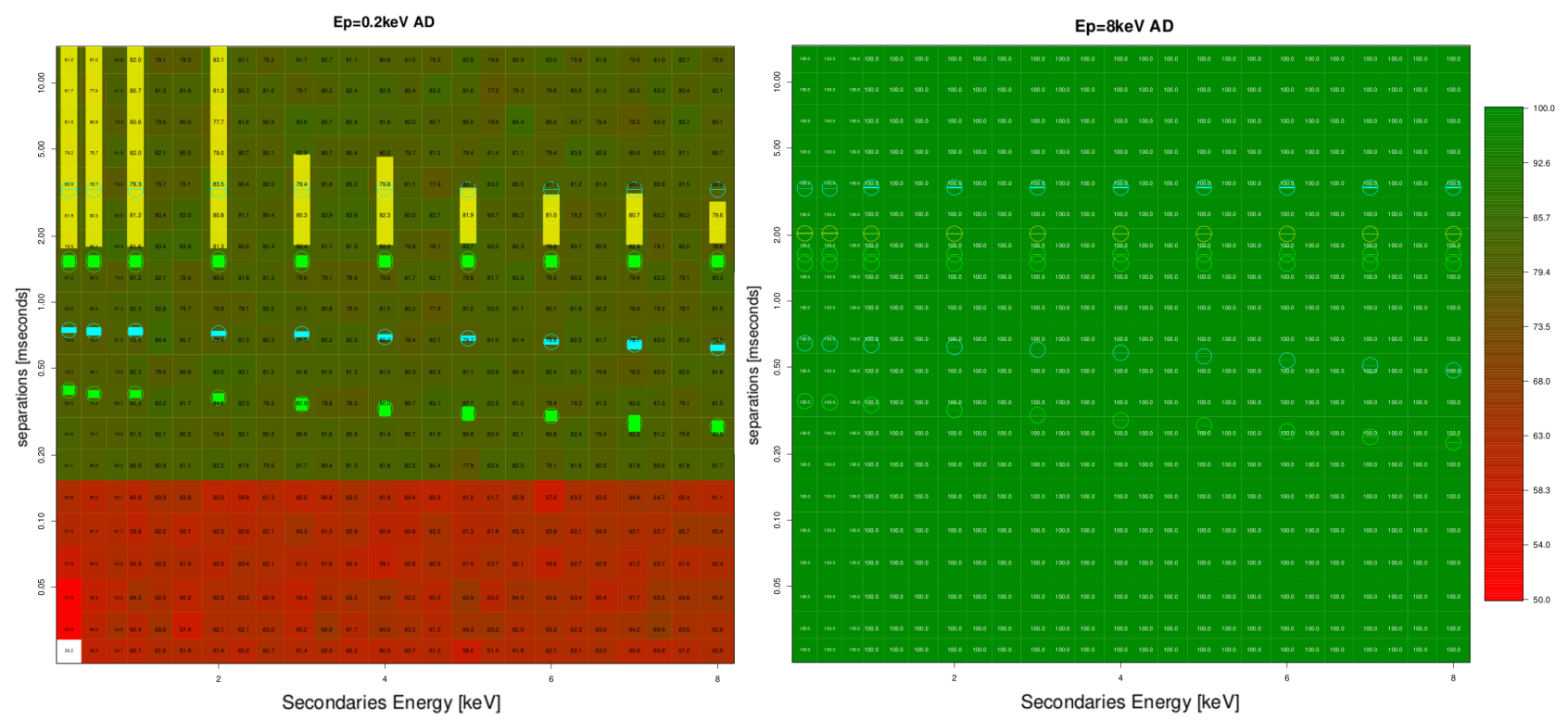

Figure 5. Detection maps of primary pulses for $\mathrm{AD}$ algorithm for $E_{P}=0.2 \mathrm{keV}$ (left) and $E_{P}=8 \mathrm{keV}$ (right). Color scales goes from $50 \%$ (red) to $100 \%$ (green). See text for details.

Analyzing the detection maps for the secondary pulses and AD (top row of Fig. 6), it can be noticed that the criteria of the slope of the derivative is hard to achieve by the smallest pulses, no matter if they are primaries or secondaries (first columns of cells in the plots of the top row). The white and reddish area in the bottom left corner means that secondary pulses are lost because of a bad subtraction between the primary pulse and the corresponding template: the small separations between primaries and secondaries and the low energy of the secondaries make the problem worse. If the energy of the secondaries increases, the detection probability improves. And if the energy of the primaries increases, the closer the pulses are, the worse the detection probability is. In the STC case (bottom row of Fig. 6), detection probability of the secondaries is always bad (white areas), or even more, it is null for small separations of the pulses, specially for the lowest energy secondary pulses. 

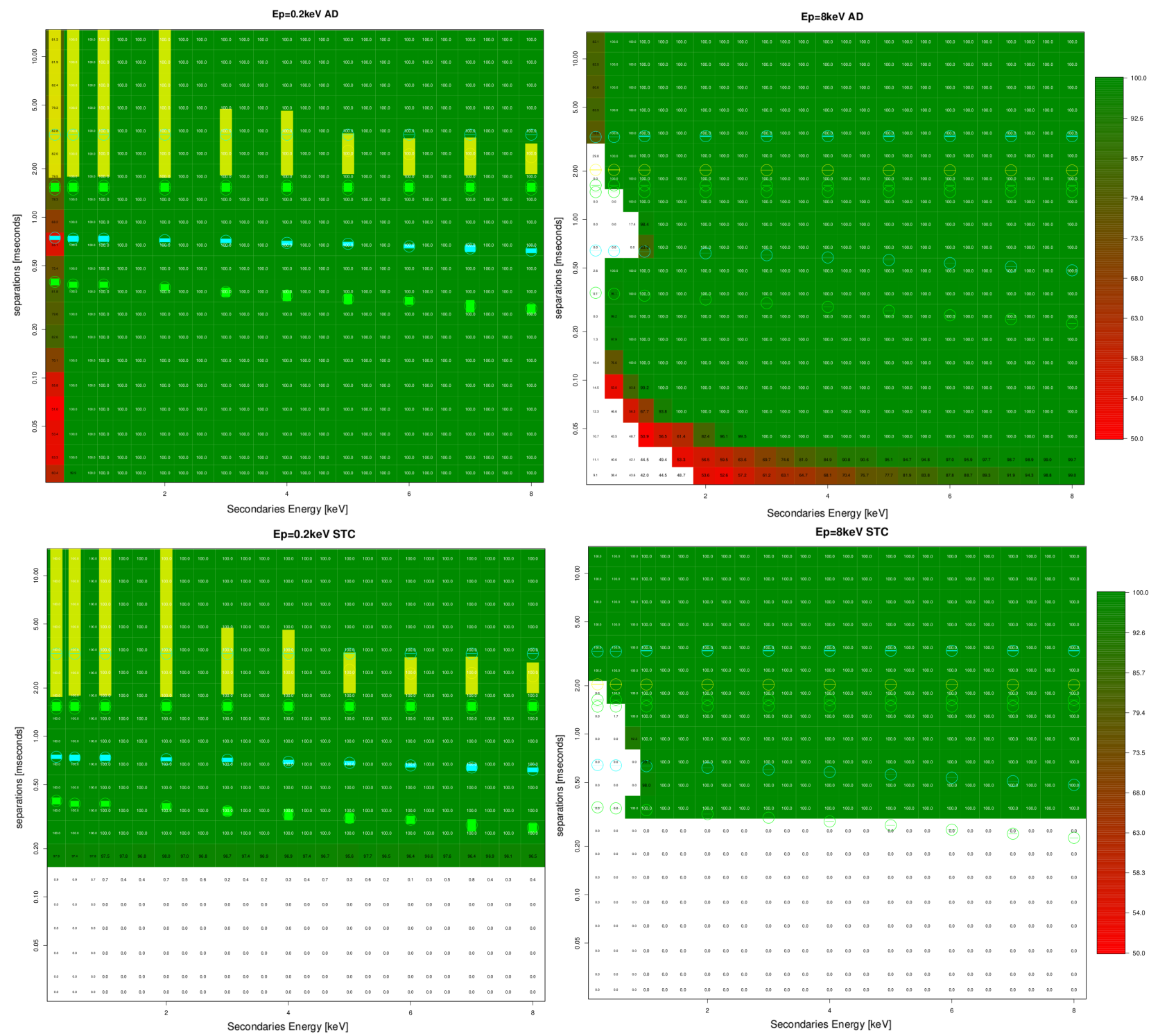

Figure 6. Detection maps of secondary pulses for AD algorithm (top row) and STC algo. (bottom row) for $E_{P}=0.2 \mathrm{keV}$ (left column) and $E_{P}=8 \mathrm{keV}$ (right column). Color scales goes from 50\%(red) to 100\%(green). See text for details.

From the analysis of these maps, we could conclude that, in terms of the detection capability, the Adjusted Derivative method has clearly a better performance than the Single Threshold Crossing mechanism. However, $\mathrm{AD}$ will loose some of the smallest pulses $(0.2 \mathrm{keV})$, both primaries and secondaries, at the smallest separations where STC seems to perform better. STC will loose many secondary pulses at all energies when they are closest to $<40 \mathrm{~ms}$ to a primary one as well as the smallest pulses $(0.2-0.5 \mathrm{keV})$ at shorter separations.

As it can be seen in the AD figures, the areas (separations/energies) where it is not possible to distinguish single pulses from piled-up pulses (fluorescent areas) overlap those areas where the detection is not good (reddish). Thus, for the AD case, flagging would not be of much help for low energy pulses lost by the detection although it could be used to flag other failed detections at larger secondary energies where the overlapping areas is smaller. STC would have less problems for the smallest primary pulses but the conflict areas would enter now the zones of larger secondaries and small separations where STC does perform worse (see circles over white cells for Es $>2$ $\mathrm{keV}$ in Fig. 6), preventing the flagging of these undetected pulses. 


\subsection{Fake maps}

Due to the imperfect subtraction of the pulse template done with the AD algorithm, there will be cases where the residuals will mimic the presence of a true pulse and could also avoid the filter imposed by the slope criteria, leading to the detection of false pulses.

With the STC approach, these false detections are less common but can also appear due to the noise in the tail of the derivative of smallest pulses. This effect could be mitigated increasing the trigger dead time when the threshold is crossed down, missing then the possible arrival of true pulses in that dead time.

In the fake detection maps, every plot gives information about the percentage of unreal detected pulses for pairs of pulses with a given primary energy. As for the detection maps, the $\mathrm{X}$ axis represents the energy of the secondary pulse $(\mathrm{keV})$ and the $\mathrm{Y}$ axis the separation of the pulses (ms). If the percentage is $0 \%$ (black numbers), no fake pulses are detected (red numbers correspond to percentages of fake pulses greater than $0 \%$ ).

In the left map of Fig. 7, representing the $\mathrm{AD}$ method and $E_{P}=0.2 \mathrm{keV}$, there are a lot of red numbers all over the plot meaning that the detection mechanism is giving some false positive pulses. For these smallest pulses an imperfect subtraction of a template after their detection, leads to fake pulses close to them (primaries or secondaries). For primaries with higher energies (right map of Fig. 7), the reddish-numbers area in the bottom right corner means that the residuals cause also non-true detections when the separations are small and the energy of the secondaries is large. Fake pulses are then located close to these large secondaries. The higher the energy of the primaries is, the more orange-colored the bottom right corner is, because there are more fake pulses in the tail of the secondaries.

In the STC case, the derivatives of the smallest pulses (primaries or secondaries) are the most sensitive to the noise. Then, some variations due to noise are detected as pulses in the tail of the smallest ones $(0.2 \mathrm{keV})$ and, in consequence, some red numbers would appear in the plot corresponding to $E_{P}=0.2 \mathrm{keV}$ and in the first columns of the other ones. Nevertheless, when the energy of the primaries or secondaries is greater than $0.2 \mathrm{keV}$, false pulses are never detected.

It is clear in any case that the STC mechanism is less sensitive to the fake detection of pulses.
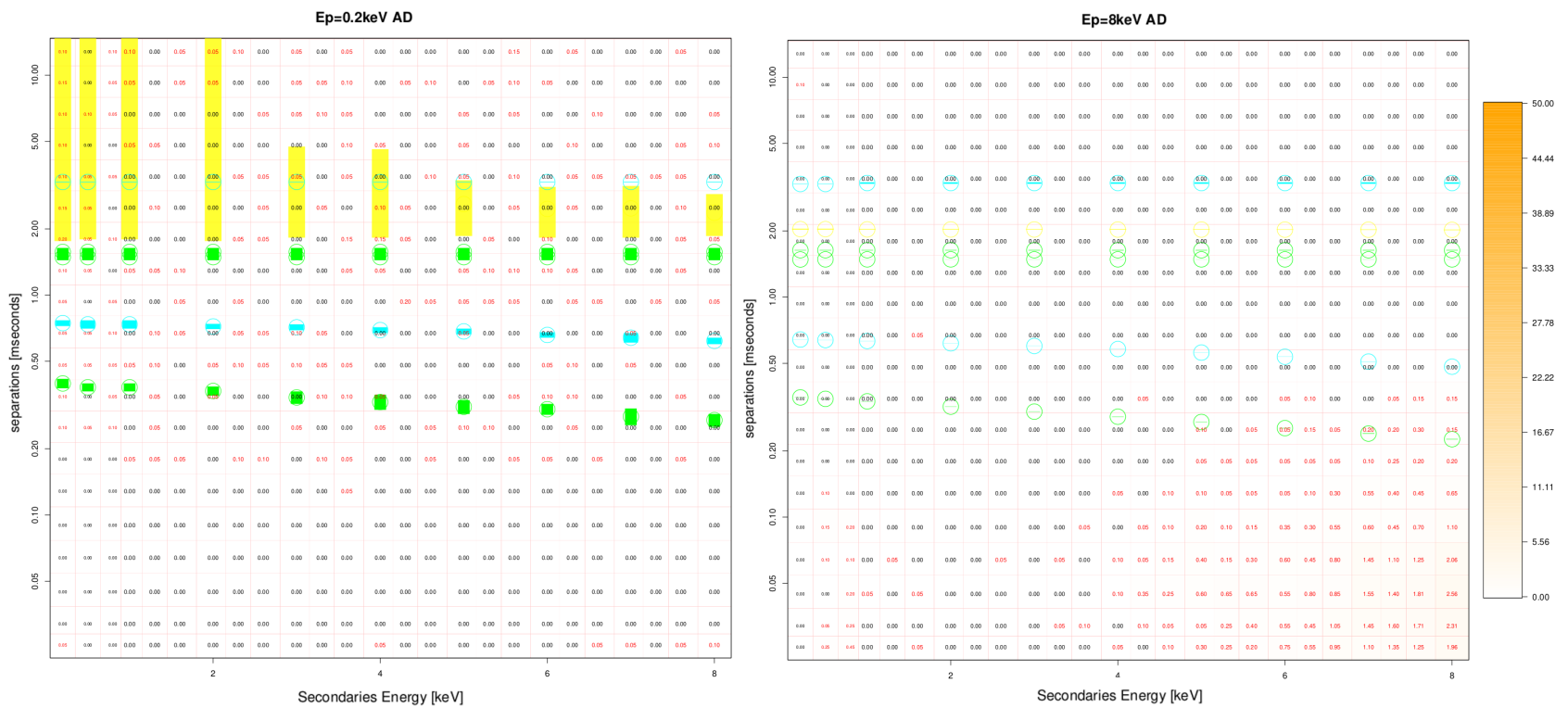

Figure 7. Fake maps for $\mathrm{AD}$ algo. for $E_{P}=0.2 \mathrm{keV}$ (left) and $E_{P}=8 \mathrm{keV}$ (right). Color scales goes from $0 \%$ (white) to $50 \%$ (orange). 


\section{FREQUENCY OF OCCURRENCE}

The frequency of occurrence of pairs of pulses (of whatever energy and separation) has been studied with simulations of the LPA2shunt array (40MUX) with a typical Crab spectrum at different fluxes (from $E=10^{-4} \mathrm{mCrab}$ to 1Crab). Photons were simulated by SIXTE/xifupipeline(http://www.sternwarte.uni-erlangen.de/research/sixte) $\left(\Gamma=2.05, N_{H}=2.16 \cdot 10^{21} \mathrm{~cm}^{-2}\right)$. Fluxes larger than $0.25 \mathrm{mCrab}$ were defocused and both Be filter and no filter scenarios have been simulated.

As it can be seen in the top plot of Fig. 8, representing the undetected fraction of photons taking into account the detection percentage of the previous detection maps, the removal of the smallest pulses done by the Be filter, produces better numbers for STC and AD. In addition, AD behaves globally better (it looses less pulses) due to the bad detection that STC does at small separations.

The bottom plot represents the undetected and unflagged fraction of photons taking into account the information provided by the detection maps and the bagplots for the conflicting area. AD produces a peak of lost pulses out of the rejection area (bagplots cannot distinguish and piled-up is produced) of around $0.01 \%$ at flux levels of $\sim 1$ Crab without the Be filter and compatible with $0 \%$ with the Be filter on. The STC mechanism peaks around $0.04 \%$, both with and without Be filter. At 1 Crab, only around $30 \%$ of these lost and unflagged pulses have small energies $(<1 \mathrm{keV})$ so the driving feature here is the behavior of both algorithms at larger energies and small separations. Looking at the detection maps for STC (bottom right map of Fig. 6), it can be seen that some of the areas where the flagging mechanism is not reliable enter the zone where STC fails for detection at larger energies and small distances between pulses. AD is less affected because detection in this zone is better. The low fraction of low energy photons among the problematic ones explains also why there is almost no difference between using Be filter or not.
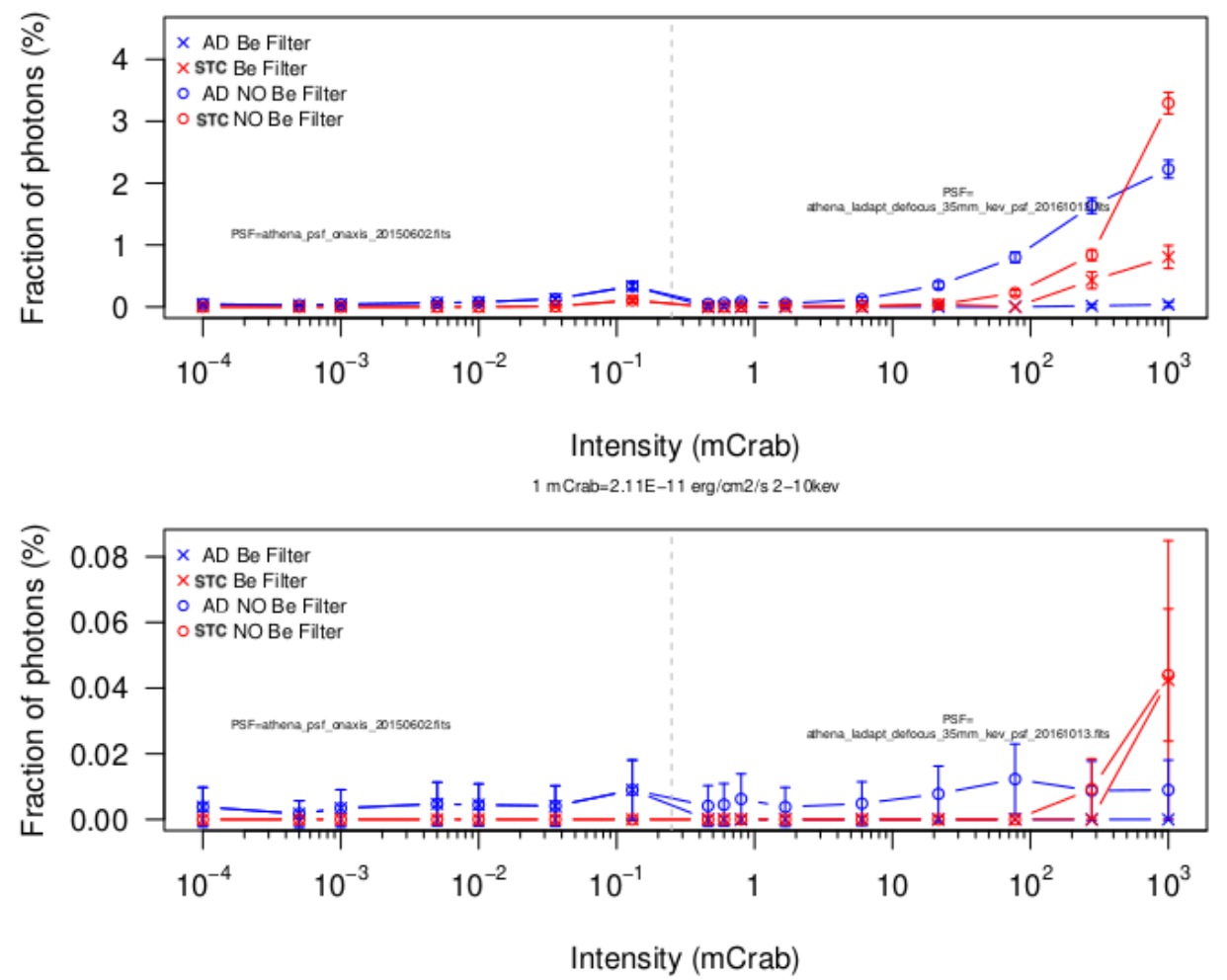

Figure 8. Top: Fraction of photons lost by the detection with AD (blue) and STC (red) algorithms using Be filter (crosses) or not (circles). Bottom: Fraction of photons lost that would produce a pileup and could not be noticed by the flagging mechanism. 
It should also be noted that an event is not flagged as pile-up, only if its high resolution estimate is compatible with its low resolution estimate. As the latter is based on the very first points of the pulses, it is effectively unaffected by the missed event and constitutes a reliable low resolution energy estimate. As a consequence, these unflagged events are more low resolution events misinterpreted as high resolution events than they are real pile-up and should only slightly deteriorate the high resolution line spread function considering they only number to at most $0.1 \%$, and only at the highest count rates. The few missed secondaries (that number up to $1 \%$ ) should also not affect the spectrum as they would have been flagged as invalid anyway in the grading process. ${ }^{11,12}$

\section{COMPUTATIONAL POWER}

In addition to the detection capability of the last two schemes, it is also relevant to analyze the consumption of computational resources in order to add an additional criteria to establish a decision framework. In this sense, a crude comparative analysis of the number of operations (additions and multiplications represented by ' + ' and ' $x$ ', respectively) made by each of the two previous alternatives is presented.

Roughly, in the Adjusted Derivative method there are some main tasks in terms of number of operations:

- finding the appropriate template (by interpolation) for the first time to make the dot product with the signal to be able to find an initial start time (Interpolation1 in Tab. 2)

- the dot product of the detected pulse with the template

- loop to choose the correct template (due to the jitter) (8 loops as maximum and 3 lags as maximum in this part)

- finding the appropriate template (by interpolation) to subtract it from the signal (Interpolation2 in Tab. 2)

- the subtraction itself

As only 25-samples-long pulses and templates are used for the dot product and 100-samples-long pulses and templates are used to subtract during the detection process, the number of additional (over the STC approach) operations is presented in Tab. 2.

Table 2. Operations invested in the AD detection.

\begin{tabular}{|c|c|c|c|}
\hline Task & Times the task occurs & + & $\mathrm{x}$ \\
\hline Interpolation1 & $1+8$ (loop) & $(1+8) \cdot 25$ & $(1+8) \cdot 2 \cdot 25$ \\
\hline Interpolation 2 & 1 & 100 & $2 \cdot 100$ \\
\hline Dot product & $5+8 \cdot 3$ (loop) & $(5+8 \cdot 3) \cdot 25$ & $(5+8 \cdot 3) \cdot 25$ \\
\hline Subtraction & 1 & 100 & \\
\hline TOTAL & & 1150 & 1375 \\
\hline
\end{tabular}

In the STC case, as neither the dot product nor the subtraction are needed, it is not necessary either to find the appropriate template. Thus, the number of the operations mentioned above will be $\sim 0$ (averaging the first 4 samples of the derivative in order to flag the pile-up is negligible).

On the other hand, it could be interesting to compare the number of operations invested in the detection process with the number of operations done in the reconstruction stage. If the reconstruction method would be optimal filtering in the resistance space ${ }^{4}$ the computational cost to process one high resolution event of $\mathrm{N}=8192$ samples would be $7 \mathrm{~N}\left(3 \mathrm{~N}^{\prime}+{ }^{\prime}+4 \mathrm{~N}^{\prime} \mathrm{x}^{\prime}\right)=57344$ operations. Therefore, in high resolution, the AD detection process would be $\sim 4.4 \%$ of the total number of operations of the detection and optimal filtering in the resistance space reconstruction scheme. 
However, there is an scenario where this fraction could be more significant. For large count rates with pulses arriving closer than the large resolution filter, shorter filters would be used for the energy reconstruction (less operations), while the detection stage would require more processing due to the larger residuals produced by the template subtraction.

\section{DIFFERENT INSTRUMENTAL SCENARIOS}

\subsection{Reduction of the number of templates}

The same study about the detection performance has been performed using for the AD algorithm half the number of calibration templates. As templates play no role in the STC detection algorithm, its performance is not affected by this approach. The rejection areas (flagging process) are also independent of the calibration templates as pulse energies are reconstructed always with the same $6 \mathrm{keV}$ filter.

Surprisingly, the performance of the AD mechanism is really similar to that in the previous case where more calibration templates were used (see Fig. 5 and top row of Fig. 6) probably pointing to a relatively small non-linearity of the simulated pulses.

\subsection{Reduced sampling rate}

The comparative study between AD and STC will be undoubtedly affected by a reduction in the sampling rate to half its baseline value (i.e. from $156.25 \mathrm{kHz}$ to $78.125 \mathrm{kHz}$ ). To evaluate these effects, the bagplot maps were also redone for pulses simulated with the reduced sampling rate and reconstructed with filters half the length of those used for the case with the baseline sampling rate.

Both the detection and fake maps applying the AD mechanism get slightly worse when the sampling rate is reduced by a factor of 2 . In the detection maps (Fig. 9), the reddish areas grow for the smallest separations meaning more pulses lost. In the fake maps (Fig. 10), the bottom right corners become more intensely orangecolored, meaning more non-true pulses. If the sampling rate decreases, the sampling time increases and the negative effects of the jitter on the detection performance become more damaging. Regarding the STC case, detection and fake maps are quite similar when the sampling rate is half its initial value although the overlap of conflict areas of pile-up confusion and undetection (reddish) area at smaller separations is lower (see Fig. 11).
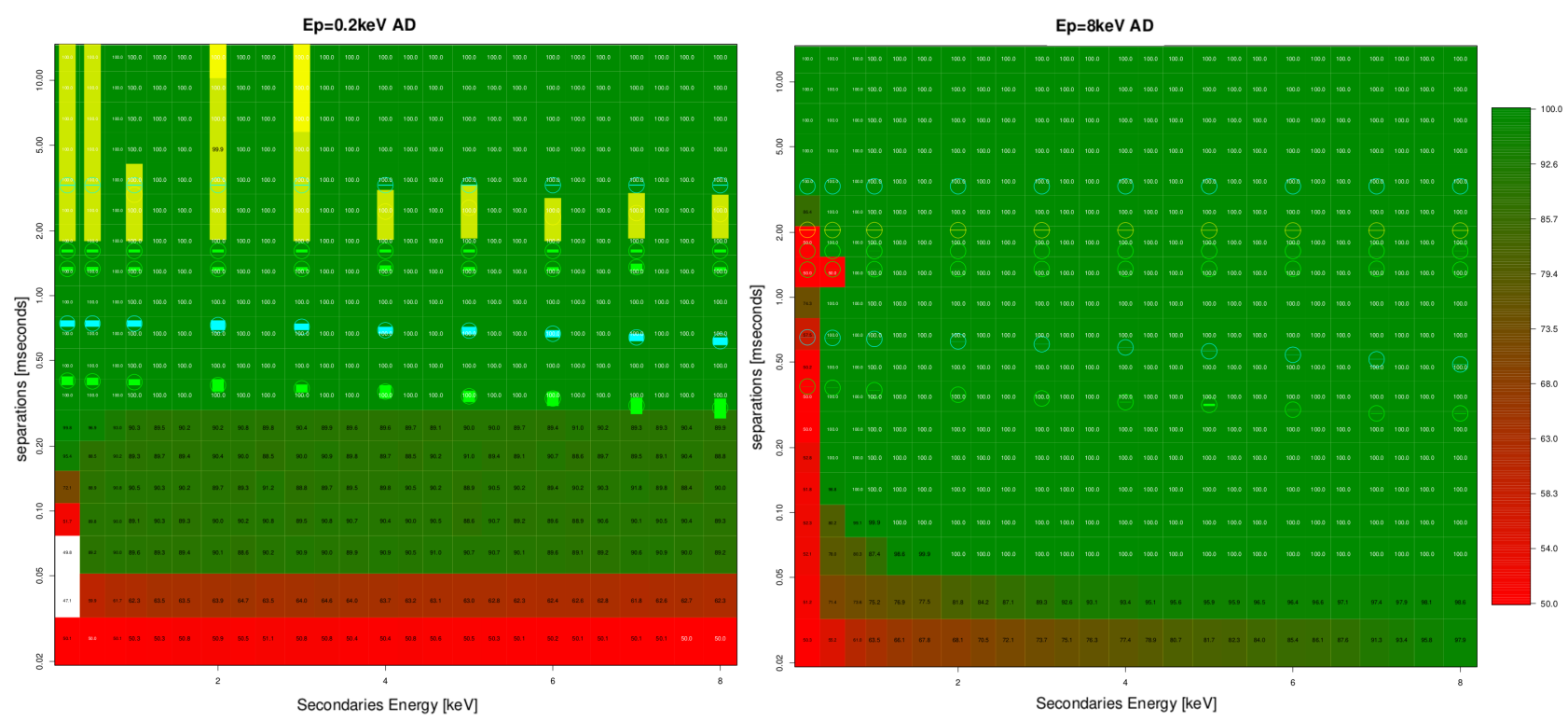

Figure 9. Detection maps $(\mathrm{P}+\mathrm{S})$ with half the sampling rate for $\mathrm{AD}$ algo. for $E_{P}=0.2 \mathrm{keV}$ (left) and $E_{P}=8 \mathrm{keV}$ (right). Color scales goes from $50 \%$ (red) to $100 \%$ (green). 

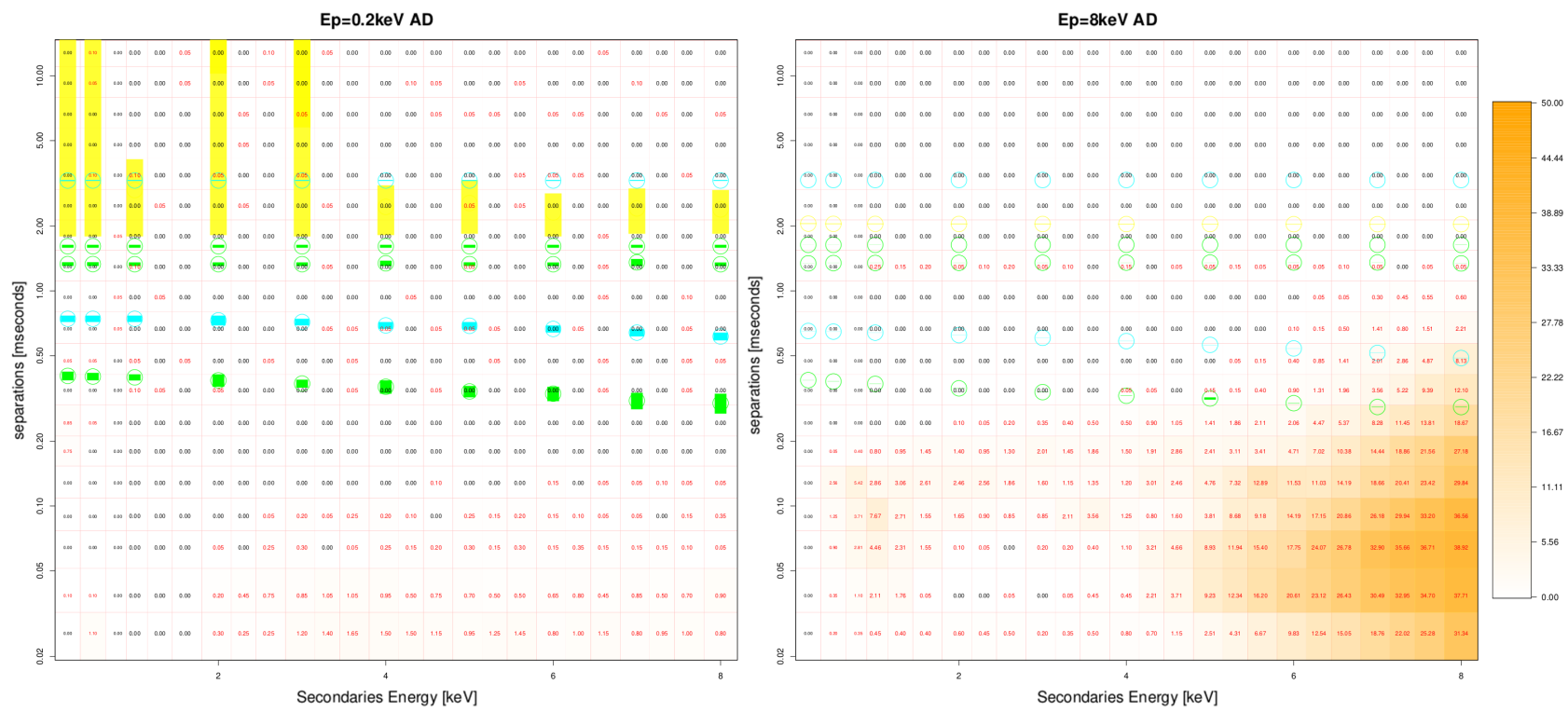

Figure 10. Fake maps with half the sampling rate for $\mathrm{AD}$ algo. for $E_{P}=0.2 \mathrm{keV}$ (left) and $E_{P}=8 \mathrm{keV}$ (right). Color scales goes from $0 \%$ (white) to $50 \%$ (orange).
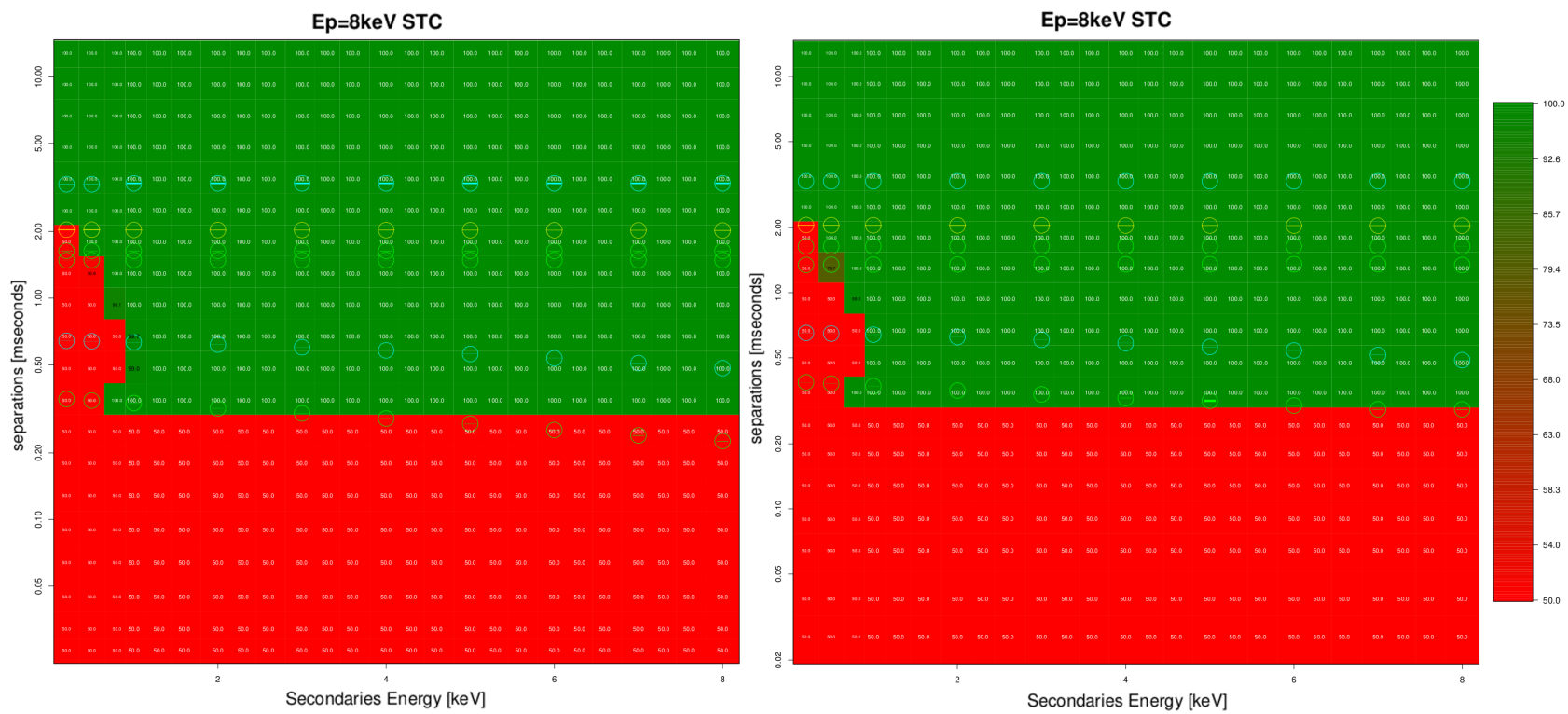

Figure 11. Detection maps $(\mathrm{P}+\mathrm{S})$ for STC algo. and $E_{P}=8 \mathrm{keV}$ with the original sampling rate (left) and with half the sampling rate (right). Color scales goes from $50 \%$ (red) to $100 \%$ (green).

Reducing the sampling rate to half its baseline values, almost does not make any difference in the fraction of undetected photons (top plot of Fig. 12) for both mechanisms. STC (no Be filter) performs slightly worse because detection also worsens for this reduced sampling rate.

With respect to the fraction of photons not detected and also not rejected by the bagplots (bottom plot of Fig. 12), again STC (with no Be filter) shows a worse behavior than AD due to its poorer detection performance.

With this reduced sampling rate, STC shows a clear difference at fluxes $\sim 1$ Crab, between the presence or absence of Be filter. The key point seems to be the area of large primaries and secondaries at small separations 
at which STC fails. In contrast with the behavior at the baseline sampling rate (see left map in Fig. 11), now the overlap of undetection and unflaggable areas is lower (see right map in Fig. 11). As for the baseline sampling rate case, the fraction of undetected pile-up events is negligible also for the reduced sampling rate when the Be filter is used even at 1 Crab fluxes.
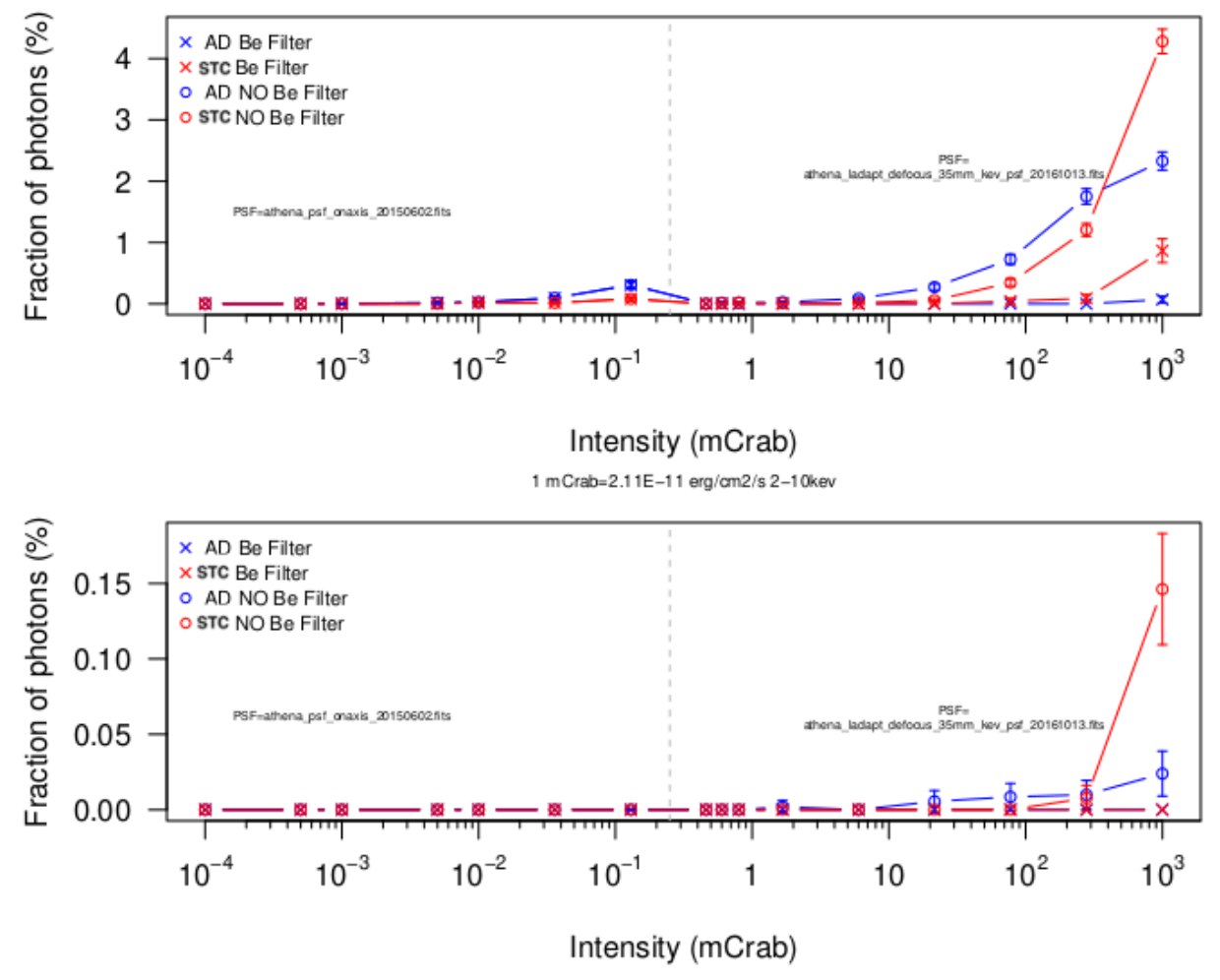

Figure 12. Top: Fraction of photons lost by the detection with AD (blue) and STC (red) algorithms using Be filter (crosses) or not (circles) with half the sampling rate. Bottom: Fraction of photons lost that would produce a pileup and could not be noticed by the flagging mechanism.

The reduction of the sampling rate will possibly affect in a significant way the results of the energy resolution. However, a further processing will be required to remove the effect of the events jitter over the energy reconstructed values. This correction is work in progress involving a $2 \mathrm{D}$ gain scale fit to obtain the calibrated energy from the phase of the photons and their reconstructed energy.

\section{CONCLUSIONS}

We have described here a comparison between two different detection algorithms that could be established as the baseline detection mechanism of the EP on board the X-IFU instrument. Even in the case of a reduced sampling rate scenario and in spite of the fact that STC has a worse detection efficiency, thanks to a post processing flagging mechanism, the fraction of undetected pile-up event stays well below $0.1 \%$ and it is expected to have negligible impact on the science data. Moreover, the reduced complexity in the computational load and calibration requirements makes the STC the preferred option as the current baseline detection algorithm of the Event Processor in the X-IFU instrument.

\section{ACKNOWLEDGMENTS}

This work has been funded by the Spanish Ministry MINECO under project ESP2016-76683-C3-1-R, co-funded by FEDER funds. 


\section{REFERENCES}

[1] Barret, D., Lam Trong, T., den Herder, J.-W., Piro, L., Barcons, X., Huovelin, J., Kelley, R., Mas-Hesse, J. M., Mitsuda, K., Paltani, S., and et al., "The Athena X-ray Integral Field Unit (X-IFU)," in [Space Telescopes and Instrumentation 2016: Ultraviolet to Gamma Ray], Proc. SPIE 9905, 99052F (July 2016).

[2] Nandra, K., Barret, D., Barcons, X., Fabian, A., den Herder, J.-W., Piro, L., Watson, M., Adami, C., Aird, J., Afonso, J. M., and et al., "The Hot and Energetic Universe: A White Paper presenting the science theme motivating the Athena+ mission," ArXiv e-prints (June 2013).

[3] Ravera, L., Cara, C., Ceballos, M. T., Barcons, X., Barret, D., Clédassou, R., Clénet, A., Cobo, B., Doumayrou, E., den Hartog, R. H., van Leeuwen, B.-J., van Loon, D., Mas-Hesse, J. M., Pigot, C., and Pointecouteau, E., "The DRE: the digital readout electronics for ATHENA X-IFU," in [Space Telescopes and Instrumentation 2014: Ultraviolet to Gamma Ray], Proc. SPIE 9144, 91445T (July 2014).

[4] Peille, P., Ceballos, M. T., Cobo, B., Wilms, J., Bandler, S., Smith, S. J., Dauser, T., Brand, T., den Hartog, R., de Plaa, J., Barret, D., den Herder, J.-W., Piro, L., Barcons, X., and Pointecouteau, E., "Performance assessment of different pulse reconstruction algorithms for the ATHENA X-ray Integral Field Unit," in [Space Telescopes and Instrumentation 2016: Ultraviolet to Gamma Ray], Proc. SPIE 9905, 99055W (July 2016).

[5] Moseley, S. H., Kelley, R. L., Schoelkopf, R. J., Szymkowiak, A. E., McCammon, D., and Zhang, J., "Advances toward high spectral resolution quantum x-ray calorimetry," IEEE Transactions on Nuclear Science 35, 59-64 (Feb 1988).

[6] Szymkowiak, A. E., Kelley, R., Moseley, S. H., and Stahle, C. K., "Signal processing for microcalorimeters," Journal of Low Temperature Physics, 93(3) , 281-285 (1993).

[7] Boyce, K. R., Audley, M. D., Baker, R. G., Dumonthier, J. J., Fujimoto, R., Gendreau, K. C., Ishisaki, Y., Kelley, R. L., Stahle, C. K., Szymkowiak, A. E., and Winkert, G. E., "Design and performance of the ASTRO-E/XRS signal processing system," in [EUV, X-Ray, and Gamma-Ray Instrumentation for Astronomy X], Proc. SPIE 3765, 741-750 (Oct. 1999).

[8] Bandler, S., Figueroa-Feliciano, E., Iyomoto, N., Kelley, R., Kilbourne, C., Murphy, K., Porter, F., Saab, T., and Sadleir, J., "Non-linear effects in transition edge sensors for x-ray detection," Nuclear Instruments And Methods In Physics Research Section A: Accelerators, Spectrometers, Detectors And Associated Equipment 559, 817-819 (April 2006).

[9] Wilms, J., Smith, S. J., Peille, P., Ceballos, M. T., Cobo, B., Dauser, T., Brand, T., den Hartog, R. H., Bandler, S. R., de Plaa, J., and den Herder, J.-W. A., "TESSIM: a simulator for the Athena-X-IFU," in [Space Telescopes and Instrumentation 2016: Ultraviolet to Gamma Ray], Proc. SPIE 9905, 990564 (July 2016).

[10] Smith, S. J., Adams, J. S., Bandler, S. R., Betancourt-Martinez, G. L., Chervenak, J. A., Chiao, M. P., Eckart, M. E., Finkbeiner, F. M., Kelley, R. L., Kilbourne, C. A., and et al., "Transition-edge sensor pixel parameter design of the microcalorimeter array for the x-ray integral field unit on Athena," in [Space Telescopes and Instrumentation 2016: Ultraviolet to Gamma Ray], Proc. SPIE 9905, 99052H (July 2016).

[11] Seta, H., Tashiro, M., Ishisaki, Y., Tsujimoto, M., Shimoda, Y., Abe, Y., Yasuda, T., Takeda, S., Asahina, M., Hiyama, Y., and et al., "The digital processing system for the soft x-ray spectrometer onboard astro-h the design and the performance," IEEE Transactions on Nuclear Science 59, 366-372 (04 2012).

[12] Peille, P. and et al., "The performance of the ATHENA X-ray Integral Field Unit," in [Space Telescopes and Instrumentation 2018: Ultraviolet to Gamma Ray], Proc. SPIE 10699 (June 2018). 